\title{
Fréquence de taille et relation taille-poids des mérous (Epinephelidae) de la pêcherie artisanale maritime ivoirienne
}

\author{
K. D. KOUASSI ${ }^{*}$, Konan N'DA et Yaya SORO \\ Laboratoire de Biologie et Cytologie Animales, UFR Sciences de la Nature (Université d'Abobo-Adjamé), \\ Abidjan 20, Côte d'Ivoire. \\ *Auteur correspondant ; E-mail : dongokoff2003@yahoo.fr 20 BP 1291 Abidjan 20.Tel : (225) 05865469
}

\section{RESUME}

Dans les eaux maritimes ivoiriennes, six espèces de mérous sont dénombrées. Ce sont Epinephelus aeneus, Epinephelus caninus, Epinephelus guaza, Epinephelus marginatus, Epinephelus alexandrinus et Epinephelus taeniops. La gamme de taille pour E. aeneus varie de $10 \mathrm{~cm}$ à $190 \mathrm{~cm}$ pour un poids total de 11 $059,8 \mathrm{~kg}$. Celle d'E. caninus et $E$. guaza est de $10 \mathrm{~cm}$ à $150 \mathrm{~cm}$ pour des poids respectifs de $5228,31 \mathrm{~kg}$ et 4 $747,63 \mathrm{~kg}$. E. marginatus se situe dans la gamme de taille de $20 \mathrm{~cm}$ à $100 \mathrm{~cm}$ avec un poids total de 1323,01 $\mathrm{kg}$. La même gamme de taille a été constatée pour E. alexandrinus et E. taeniops, soit $30 \mathrm{~cm}$ à $110 \mathrm{~cm}$. Les poids respectifs correspondants sont de $416,3 \mathrm{~kg}$ et $170,45 \mathrm{~kg}$. Pour toutes ces espèces, le mode est situé dans la classe [40-90] cm. La relation qui lie la longueur à la masse est de type allometrique minorante pour les cinq premières espèces et allometrique majorante pour la dernière espèce. Il existe une forte corrélation entre le poids total et la longueur totale pour les cinq premières espèces et entre le poids total et la longueur à la fourche pour Epinephelus alexandrinus comme en témoignent les coefficients de corrélation $(\mathrm{r}=0,89$ pour Epinephelus aeneus, $\mathrm{r}=0,90$ pour Epinephelus caninus, $\mathrm{r}=0,87$ pour Epinephelus guaza, $\mathrm{r}=0,82$ pour Epinephelus marginatus, $\mathrm{r}=0,90$ pour Epinephelus alexandrinus et $\mathrm{r}=0,73$ pour Epinephelus taeniops). Les coefficients d'allometrie sont $: \mathrm{b}=2,92$ pour Epinephelus aeneus $(\mathrm{N}=3279$ poissons), $\mathrm{b}=2,83$ pour Epinephelus caninus ( $\mathrm{N}=1333$ poissons), $\mathrm{b}=2,81$ pour Epinephelus guaza $(\mathrm{N}=1145$ poissons $), \mathrm{b}=2,92$ pour Epinephelus marginatus $(\mathrm{N}=685$ poissons), $\mathrm{b}=2,88$ pour Epinephelus alexandrinus $(\mathrm{N}=176$ poissons $)$ et $\mathrm{b}=3,11$ pour Epinephelus taeniops $(\mathrm{N}=116$ poissons $)$.

(c) 2010 International Formulae Group. All rights reserved.

Mots clés : Allometrie, mérous, pêche artisanal, Côte d'Ivoire.

\section{INTRODUCTION}

L'un des problèmes auxquels sont confrontés les pays en voie de développement est l'insuffisance des denrées alimentaires d'origine animale aussi bien qualitativement que quantitativement. Une fraction importante de ce besoin est couverte par le poisson, issu de la pêche (industrielle et artisanale).

Le mérou est l'un des poissons le plus connu qui se rencontre le long des côtes européennes, africaines et des côtes méditerranéennes (Hureau, 1986). Il 
appartient à la famille des Serranidae et au genre Epinephelus. Il est devenu une espèce protégée, car menacée d'extinction en France (GEM, 2006).

En Afrique et singulièrement en Côte d'Ivoire, sa capture est devenue fragmentaire. Même la pêche à des saisons censées être fructueuses est devenue catastrophique. Sa rareté au cours des marées et la variabilité des gammes de taille enregistrées amènent à poser la problématique de sa pérennité.

L'objectif général de ce travail est de faire une contribution à la maîtrise des facteurs de variation du stock des Mérous en Côte d'Ivoire. Plus spécifiquement, il permettra de mettre en exergue l'évolution des gammes de taille pêchées et d'établir les équations taille-poids correspondantes afin d'en ressortir les différentes corrélations. Ainsi, saura t-on si ces espèces exploitées ont leur pérennité menacée ou pas afin de continuer de façon durable, à l'approvisionnement de la population en protéines animales. Cette étude est réalisée entre janvier 2006 et décembre 2007.

\section{MATERIEL ET METHODES Matériel}

Il comprend le matériel biologique et le matériel technique. Le matériel biologique est uniquement composé des six espèces de mérou. Quant au matériel technique, il comprend un décamètre de longueur de dix mètres, gradué au millimètre près, une première balance est de type TIGER, de capacité $20 \mathrm{~kg}( \pm 0,05$ $\mathrm{kg}$ ), servant à peser les poissons de moins de $20 \mathrm{~kg}$. La deuxième balance est une bascule de type T001. Sa capacité maximale est de $120 \mathrm{Kg}$ et la minimale de $10 \mathrm{Kg}$. Elle sert à peser les poissons de plus de $20 \mathrm{~kg}$ et les caisses à poissons. La caisse à poisson est rectangulaire et faite en bois. Sa longueur est de $77 \mathrm{~cm}$ et sa largeur de $45 \mathrm{~cm}$. Elle a une épaisseur de $2 \mathrm{~cm}$.

\section{Méthodes d'étude \\ Mesures et prélèvements}

De janvier 2006 à décembre 2007, des échantillonnages journaliers sont effectués du lundi au samedi, parfois même les dimanches au quai artisanal du port de pêche, principal lieu de débarquement et de vente du mérou. Le débarquement s'opère de 05 heures du matin à 10 heures. Les poissons sont pêchés le long du littoral ivoirien (Figure 1). Les zones de pêche sont le sud-est précisément de la région d'Assinie à la frontière ivoiro-ghanéenne et le sud-ouest de Sassandra à la frontière Côte d'IvoireLiberia.

Sur le quai artisanal du port de pêche d'Abidjan, il est procédé à la reconnaissance des différentes espèces par les clés d'identification (description morphologique) de Wolfgang (1990), Opic et Seret (1981) et Fischer et al. (1981), puis à la mensuration et à la pesée de tous les individus des caisses débarquées si celles-ci ne sont pas nombreuses (une à deux caisses). Dans le cas contraire, un échantillon de caisse est prélevé pour ces mesures. La longueur totale $\left(\mathrm{L}_{\mathrm{t}}\right)$ du poisson est mesurée, c'est-àdire de l'extrémité de la nageoire caudale à l'extrémité de la mâchoire inférieure avec le décamètre gradué au millimètre 
près. Le poids total $\left(\mathrm{P}_{\mathrm{t}}\right)$ est obtenu à l'aide des deux balances (TIGER et bascule). Au total, 3279 E. aeneus ont été mesurés dans la fourchette de $10 \mathrm{~cm}$ à $190 \mathrm{~cm}$, pour un poids total de $11059,795 \mathrm{~kg}$. $E$. caninus et $E$. guaza ont enregistré chacun 1333 individus pour un poids de 5 228,31 $\mathrm{kg}$ et 1145 individus pour $4747,63 \mathrm{~kg}$, dans un même intervalle de taille $(10 \mathrm{~cm}$ à $150 \mathrm{~cm}) .685$ individus ont été mesurés au compte d'E. marginatus, dans une gamme de taille de $20 \mathrm{~cm}$ à $100 \mathrm{~cm}$ et pour un poids total de $1323,01 \mathrm{~kg}$. Quant à $E$. alexandrinus et E. taeniops, l'intervalle commun de capture est $30 \mathrm{~cm}-110 \mathrm{~cm}$ pour des effectifs et poids correspondants de 176 individus pour $416,3 \mathrm{~kg}$ et 116 individus pour $170,45 \mathrm{~kg}$.

\section{Exploitation des données \\ Fréquence de taille}

Le logiciel FiSAT II a permis de traiter les longueurs mesurées en histogrammes de fréquences de taille sur les deux années d'échantillonnage. Pour chaque espèce d'Epinephelus, les histogrammes normaux et ceux issus des données restructurées ont été construits. Les restructurées permettent de bien apprécier les dispositifs des histogrammes et de dégager aisément la gamme de taille la plus pêchée.

\section{Relation taille-poids}

Les données recueillies ont permis de montrer la corrélation entre la longueur et le poids. D'après Le Cren (1951), cette relation est de la forme :

$\mathrm{P}=\mathrm{aL}^{\mathbf{b}}$.

$\mathrm{P}=$ poids total du poisson en $(\mathrm{g})$,

$\mathrm{L}=$ longueur totale du poisson en $\mathrm{cm}$, $\mathrm{a}=$ constante qui est l'ordonnée à l'origine,

$\mathrm{b}=$ coefficient d'allométrie caractéristique de chaque espèce. Ce taux d'allométrie varie de 2 à 4 , mais le plus souvent est proche de 3 (N'da et al., 2006 ; Layachi et al., 2007).

Les paramètres $\mathrm{a}$ et $\mathrm{b}$ ont été calculés à partir des couples de valeurs longueurpoids par l'analyse de la variance (ANOVA) à un facteur, contenue dans le logiciel Statistica, version 7.1. La valeur du coefficient d'allométrie détermine le type de croissance :

$\mathrm{b}=3$, la croissance est isométrique, donc la densité spécifique du poisson ne change pas,

$\mathrm{b}<3$, la croissance est allométrique minorante, c'est-à-dire que le poisson s'allonge plus vite qu'il ne grossit.

b>3, l'allométrie est majorante, c'est-àdire que le poisson grossit plus vite qu'il ne s'allonge.

$\mathrm{La}$ fonction «analyse de corrélation» et plus précisément le coefficient de Pearson, du même logiciel Statistica a été utilisé pour mettre en évidence les différents liens.

\section{RESULTATS}

\section{Structure des tailles de la population des mérous}

Les Figures 2 à 7 illustrent les fréquences des tailles des espèces de mérous.

L'analyse de la composition démographique montre des intervalles de capture variés avec une dominance de la classe de taille $[35 \mathrm{~cm}-90 \mathrm{~cm}$ ] pour toutes les espèces. 
Tableau 1 : Paramètres de la relation longueur-poids des espèces de mérou pêchées sur le littoral ivoirien (traitement des données avec le logiciel Statistica 7.1).

\begin{tabular}{llllllll}
\hline espèce & $\mathbf{a}$ & $\mathbf{b}$ & $\mathbf{R}^{\mathbf{2}}$ & $\mathbf{r}$ & $\mathbf{p}$ & $\mathbf{N}$ & équations \\
\hline E. aeneus & 0,02 & 2,92 & 0,7990 & 0,89 & $\mathrm{P}<0,05$ & 3279 & $\mathrm{P}_{\mathrm{t}}=0,02 \mathrm{xL}_{\mathrm{t}}{ }^{2,92}$ \\
E. caninus & 0,03 & 2,83 & 0,8107 & 0,90 & $\mathrm{P}<0,05$ & 1333 & $\mathrm{P}_{\mathrm{t}}=0,03 \mathrm{xL}_{\mathrm{t}}{ }^{2,83}$ \\
E. guaza & 0,03 & 2,81 & 0,7551 & 0,87 & $\mathrm{P}<0,05$ & 1145 & $\mathrm{P}_{\mathrm{t}}=0,03 \mathrm{xL}_{\mathrm{t}}{ }^{2,81}$ \\
E. marginatus & 0,02 & 2,92 & 0,6684 & 0,82 & $\mathrm{P}<0,05$ & 685 & $\mathrm{P}_{\mathrm{t}}=0,02 \mathrm{xL}_{\mathrm{t}}{ }^{2,92}$ \\
E. alexandrinus & 0,02 & 2,88 & 0,8135 & 0,90 & $\mathrm{P}<0,05$ & 176 & $\mathrm{P}_{\mathrm{t}}=0,02 \mathrm{xL}_{\mathrm{f}}{ }^{2,88}$ \\
E. taeniops & 0,01 & 3,11 & 0,5362 & 0,73 & $\mathrm{P}<0,05$ & 116 & $\mathrm{P}_{\mathrm{t}}=0,01 \mathrm{xL}_{\mathrm{t}}{ }^{3,11}$ \\
\hline
\end{tabular}

$\mathrm{P}_{\mathrm{t}}=$ poids total du poisson en $(\mathrm{g}), \mathrm{L}_{\mathrm{t}}=$ longueur totale du poisson en $\mathrm{cm}, \mathrm{L}_{\mathrm{f}}=$ longueur à la fourche, $\mathrm{a}=$ constante qui est l'ordonnée à l'origine, $\mathrm{b}=$ coefficient d'allometrie caractéristique de chaque espèce, $\mathrm{r}=$ coefficient corrélation, $\mathrm{p}=$ probabilité de lien, $\mathrm{N}=$ nombre d'individus.

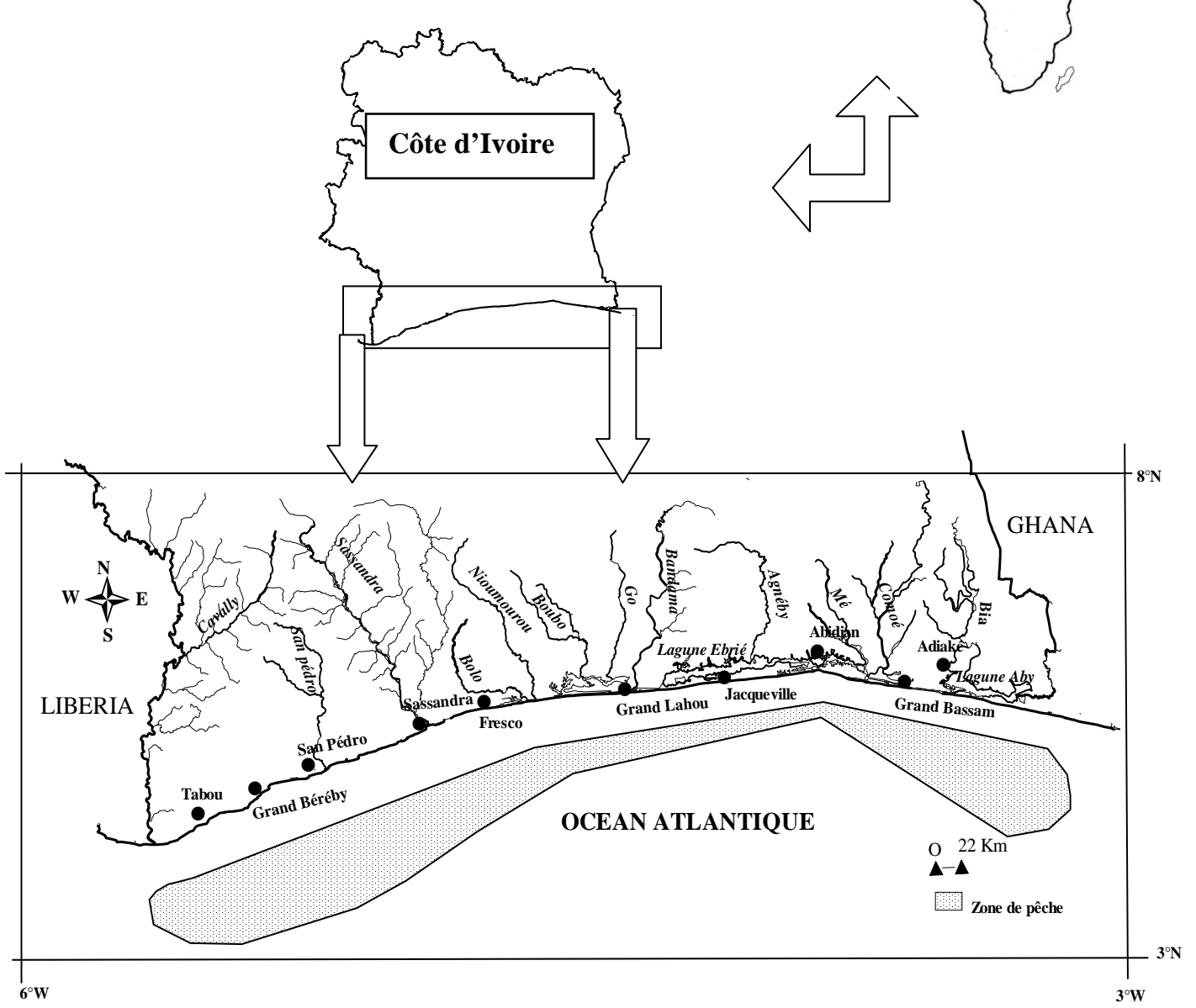

Figure 1 : Zone de pêche des mérous (Epinephelidae) sur le littoral ivoirien avec Abidjan le principal lieu de débarquement. 


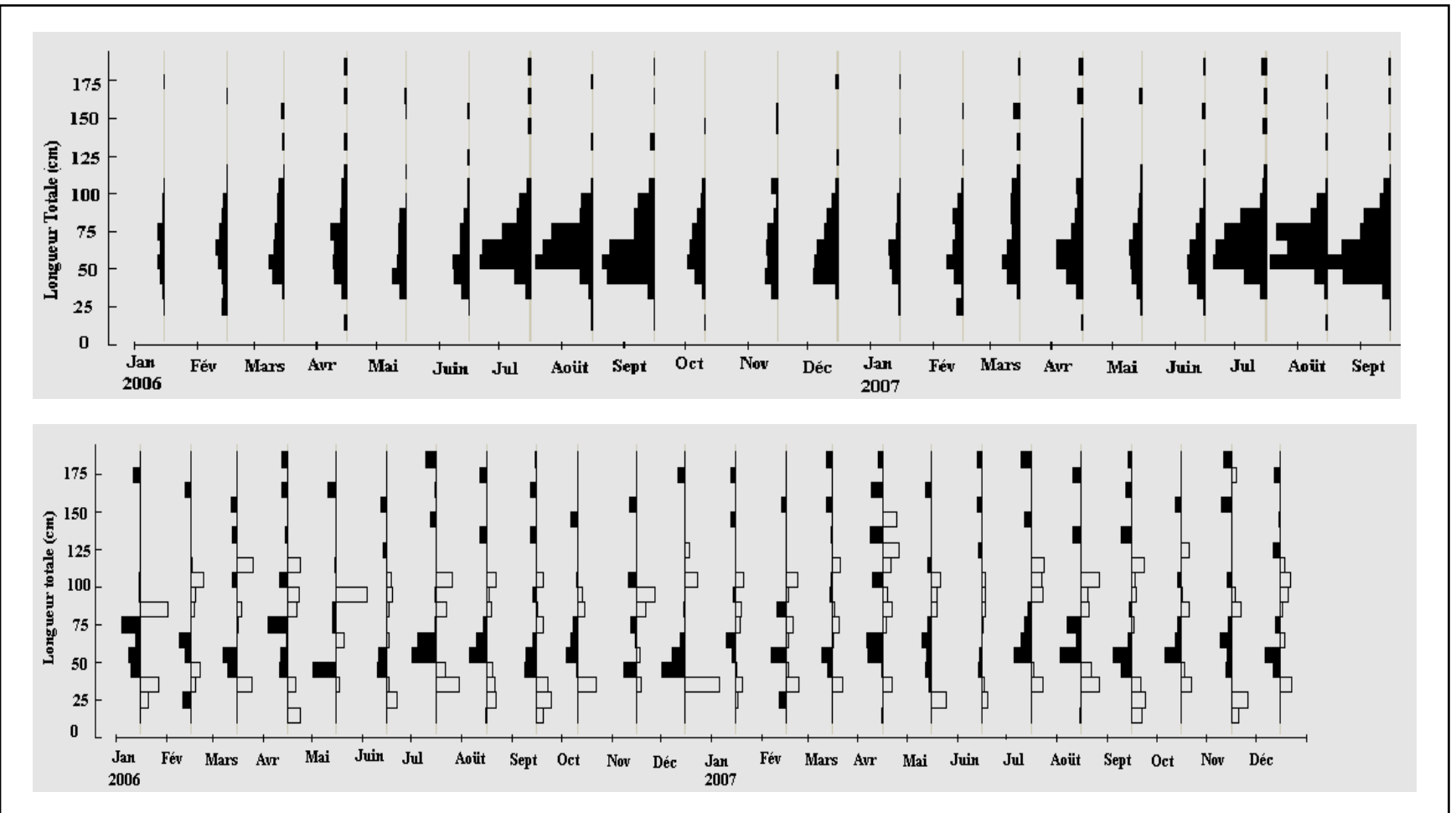

Figure 2 : Distribution de fréquence de taille chez Epinephelus aeneus (E. Geoffroy Saint-Hilaire 1817), (a) histogramme de fréquence de taille normale, (b) histogramme de fréquence de taille restructurée (les bandes noires et blanches constituent les déviations positives et négatives). 
K. D. KOUASSI et al. / Int. J. Biol. Chem. Sci. 4(3): 757-769, 2010

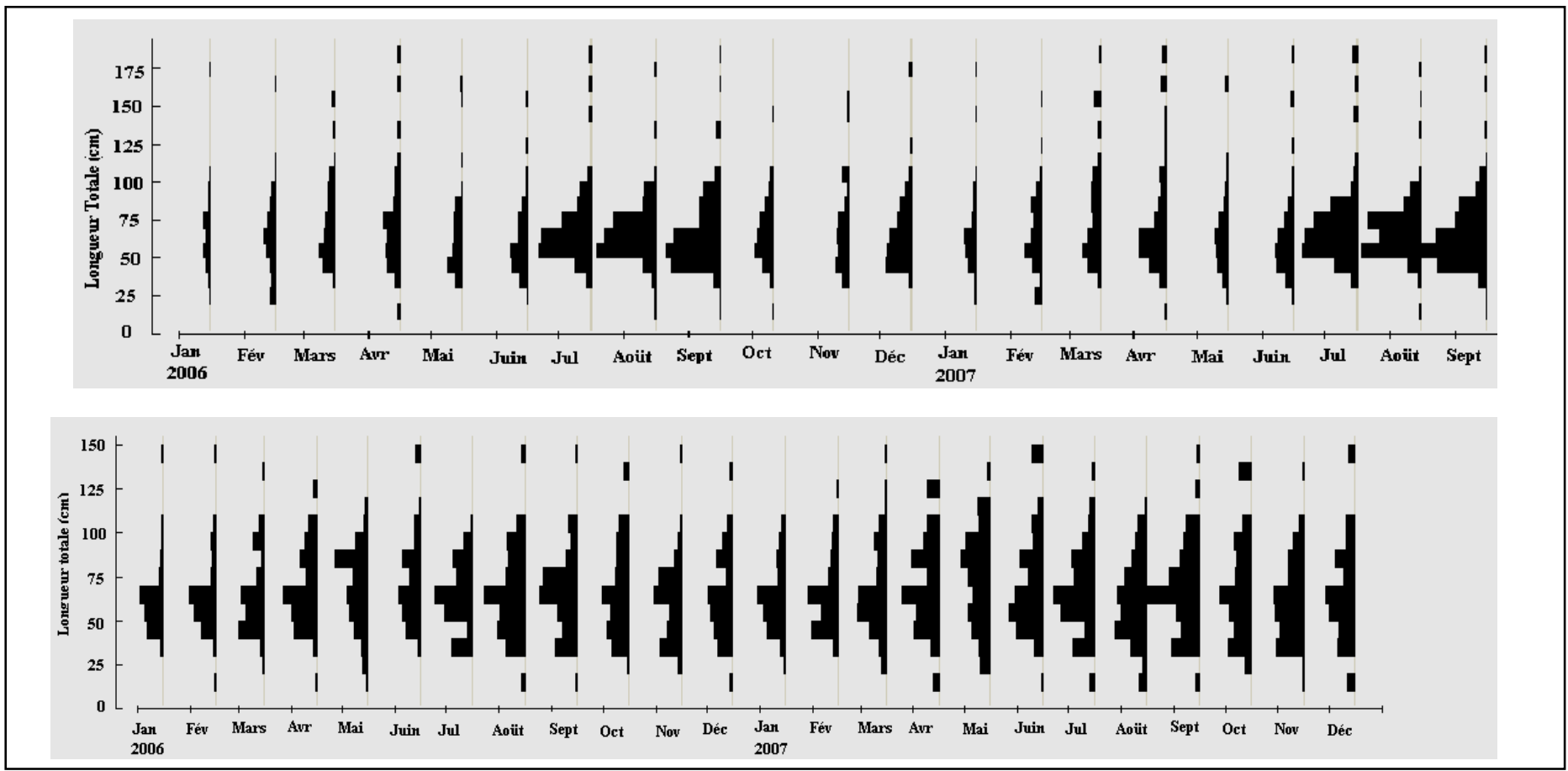

Figure 3 : Distribution de fréquence de taille chez Epinephelus caninus (), (a) histogramme de fréquence de taille normale, (b) histogramme de fréquence de taille restructurée (les bandes noires et blanches constituent les déviations positives et négatives). 


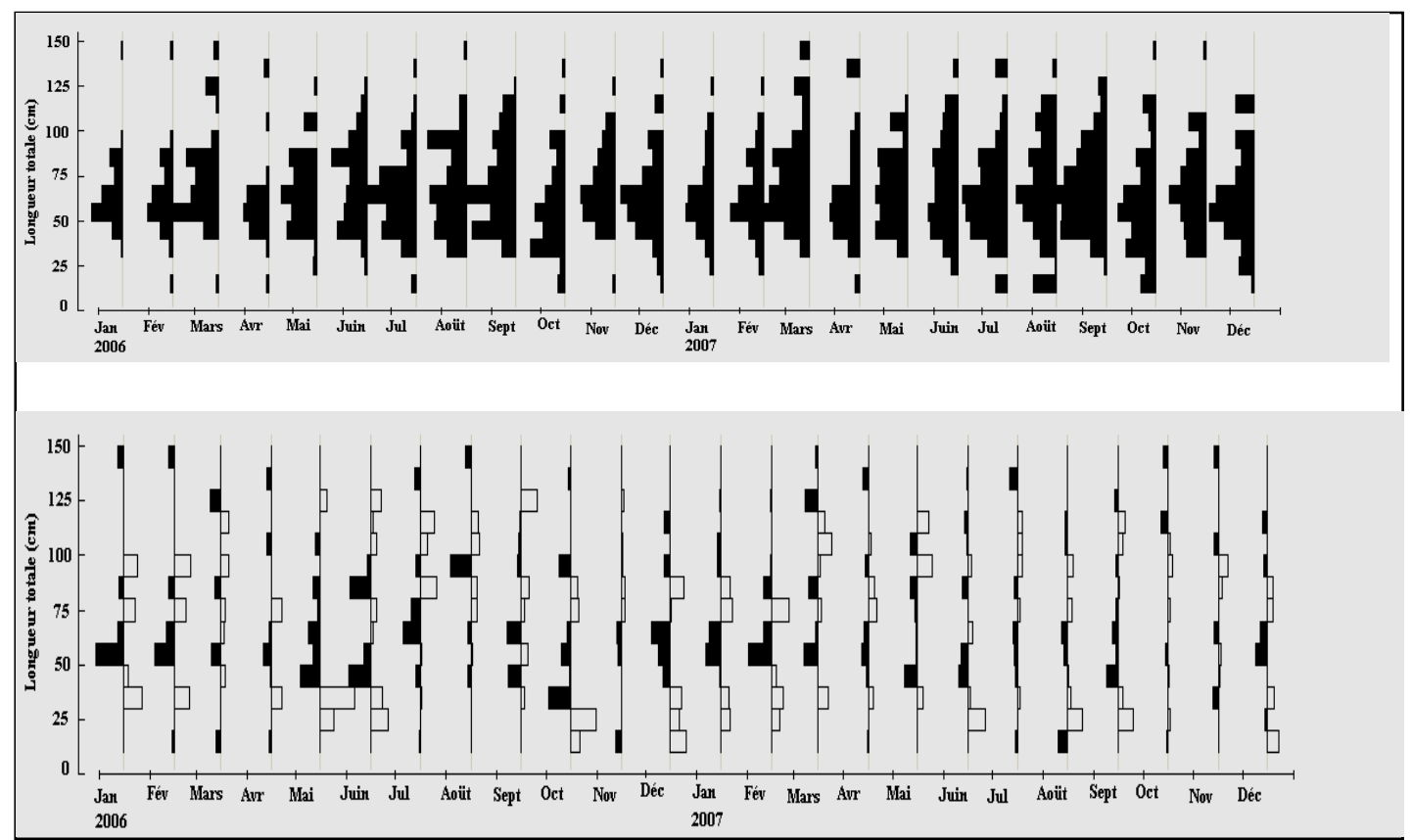

Figure 4 : Distribution de fréquence de taille chez Epinephelus guaza (), (a) histogramme de fréquence de taille normale,

(b) histogramme de fréquence de taille restructurée (les bandes noires et blanches constituent les déviations positives et négatives). 


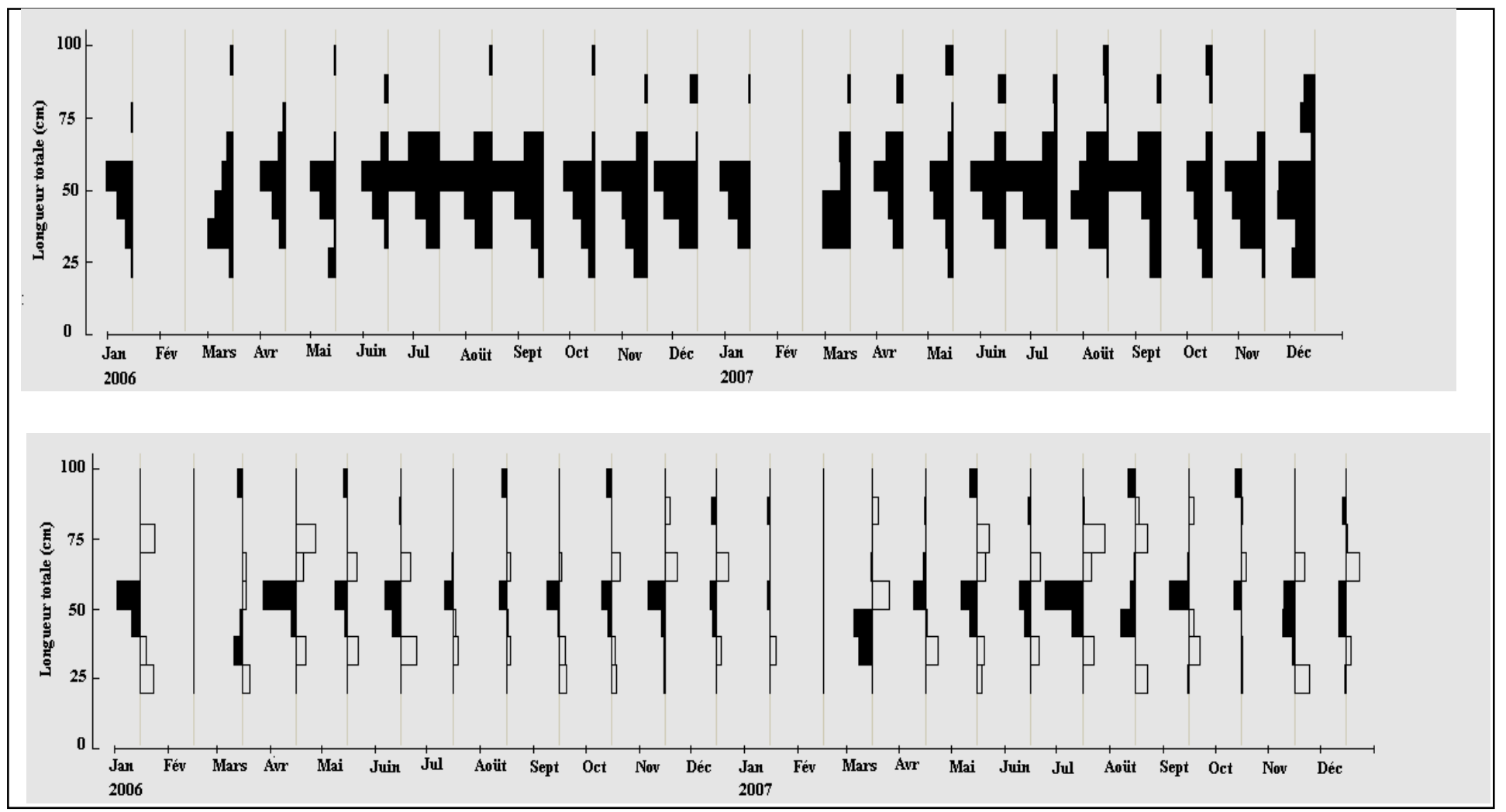

Figure 5 : Distribution de fréquence de taille chez Epinephelus marginatus, (a) histogramme de fréquence de taille normale, (b) histogramme de fréquence de taille restructurée (les bandes noires et blanches constituent les déviations positives et négatives). 


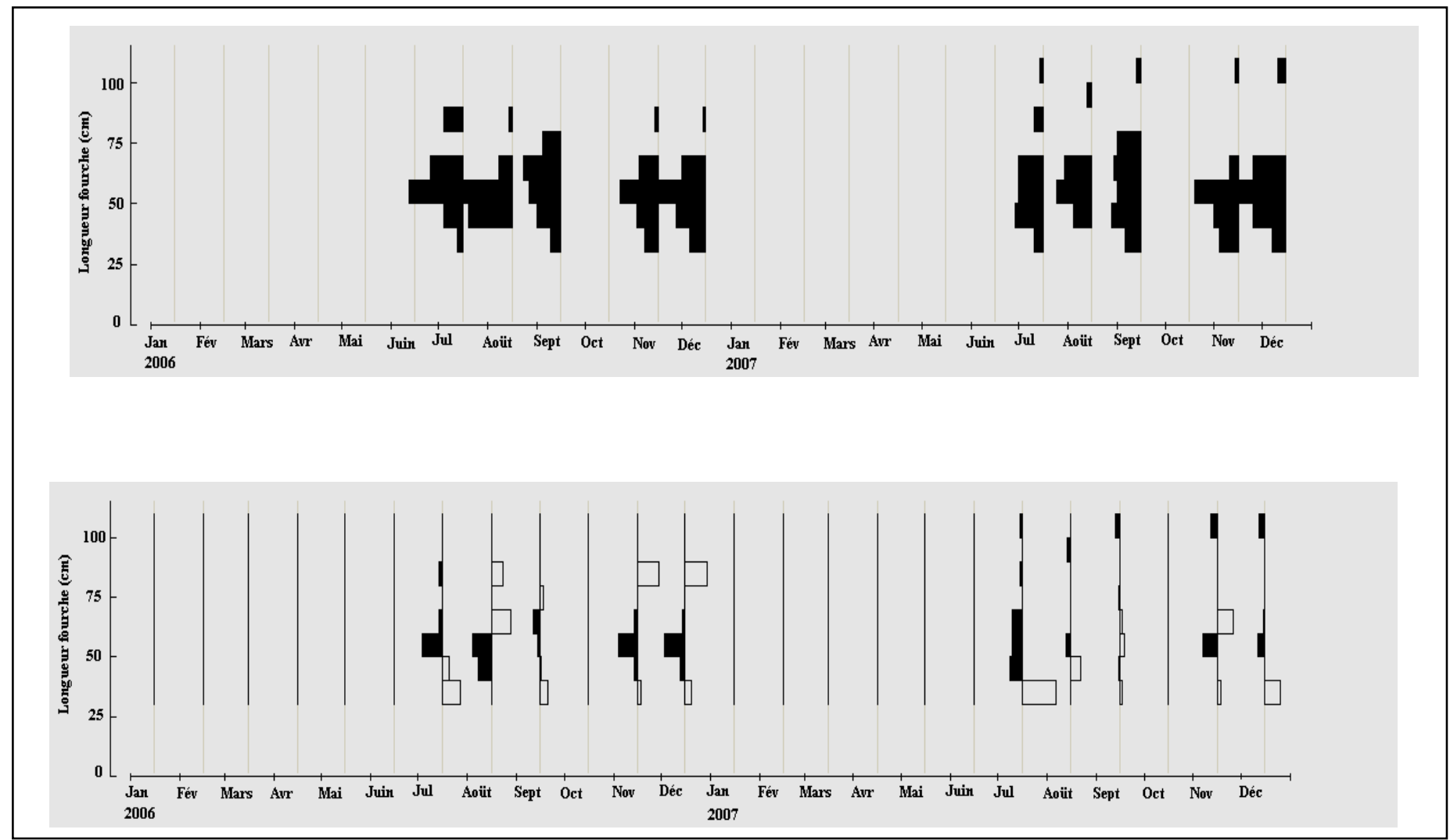

Figure 6 : Distribution de fréquence de taille chez Epinephelus alexandrinus, (a) histogramme de fréquence de taille normale, (b) histogramme de fréquence de taille restructurée (les bandes noires et blanches constituent les déviations positives et négatives). 
K. D. KOUASSI et al. / Int. J. Biol. Chem. Sci. 4(3): 757-769, 2010

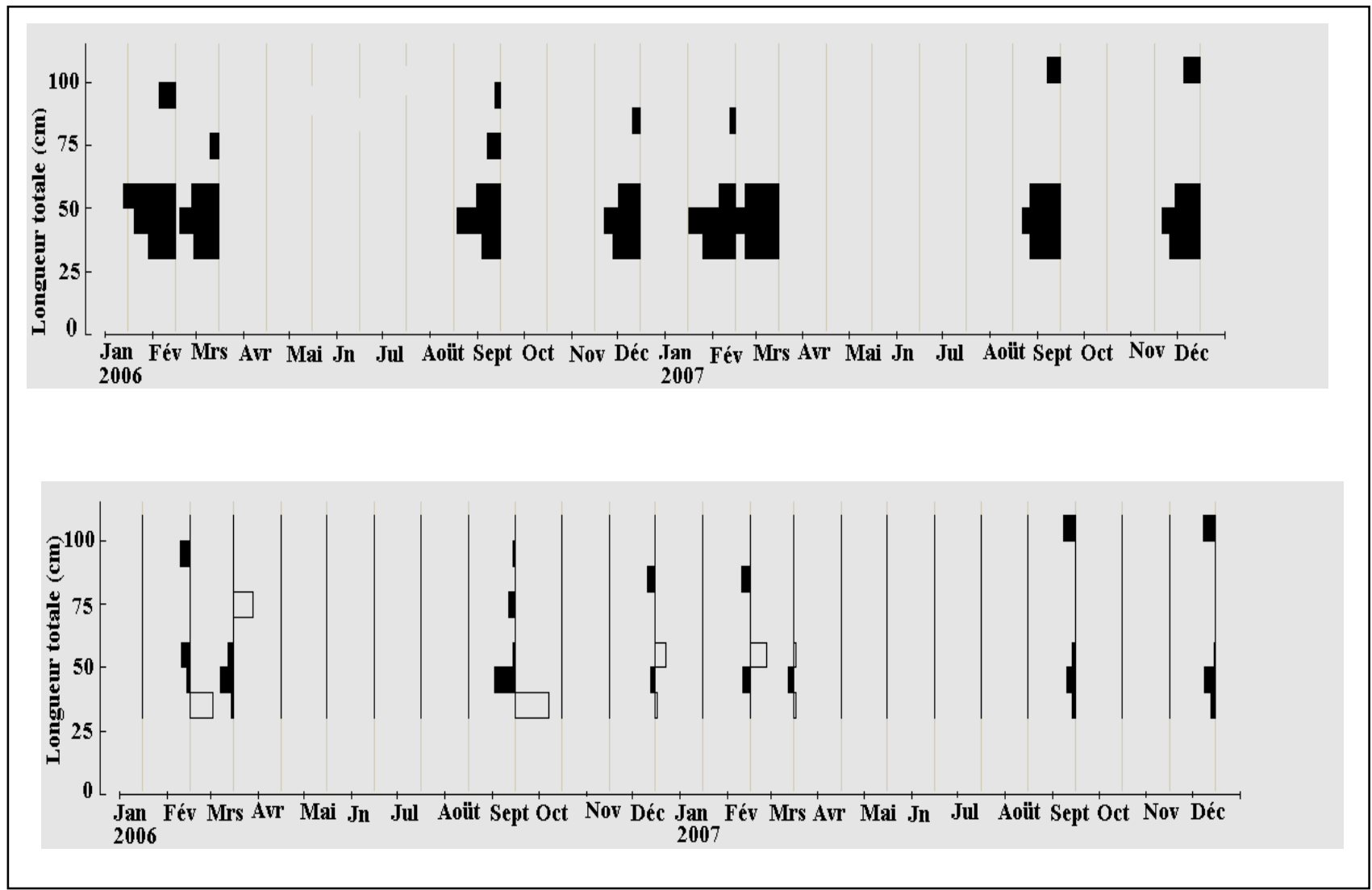

Figure 7 : Distribution de fréquence de taille chez Epinephelus taeniops, (a) histogramme de fréquence de taille normale,

(b) histogramme de fréquence de taille restructurée (les bandes noires et blanches constituent les déviations positives et négatives). 
La gamme de taille d'E. aeneus a varié de $10 \mathrm{~cm}$ à $190 \mathrm{~cm}$. Le nombre d'individus échantillonnés est de 3279 pour un poids total de $11059,8 \mathrm{~kg}$. Celle d'E. caninus et d'E. guaza est de $10 \mathrm{~cm}$ $150 \mathrm{~cm}$. Les effectifs s'élèvent à 1333 et 1145, Quant aux poids totaux, ils sont de $5228,3 \mathrm{~kg}$ et $4747,6 \mathrm{~kg}$. La plus grande taille d'E. marginatus, a été $100 \mathrm{~cm}$ et la plus petite $20 \mathrm{~cm}$. Le poids total enregistré a été $1323 \mathrm{~kg}$. Au niveau d' $E$. alexandrinus et d'E. taeniops, les tailles pêchées étaient comprises entre $30 \mathrm{~cm}$ et $100 \mathrm{~cm}$. Les poids correspondants étaient respectivement de $416,3 \mathrm{~kg}$ et $170,5 \mathrm{~kg}$. Il n'a été enregistré aucun débarquement dans les mois de janvier et de février pour ces deux espèces. Il en a été de même pour E. alexandrinus dans les mois de mars, avril, mai et juin.

Beaucoup d'individus ont été pêchés chez toutes les espèces durant les mois de juillet, août, septembre et octobre (grande saison froide).

Dans l'ensemble, chez toutes les espèces, la gamme de taille dominante, c'est-à-dire la plus pêchée, est comprise entre $35 \mathrm{~cm}$ et $90 \mathrm{~cm}$.

\section{Relations taille-poids des différentes espèces de mérou}

Pour ces six espèces de mérou, les paramètres calculés sont résumés dans le Tableau 1. Les coefficients d'allométrie des cinq premiers mérous sont inférieurs à trois. Ce qui signifie que ces espèces s'allongent vite qu'elles ne grossissent. Quant à E. taeniops, le paramètre $\mathrm{b}(3,11)$ est supérieur à trois, donc il grossit plus vite qu'il ne s'allonge.
Il existe de fortes corrélations entre les poids et les différentes tailles correspondantes car toutes les valeurs de $r$ sont élevées.

\section{DISCUSSION}

Les mérous sont des espèces hermaphrodites protogynes, c'est-à-dire qu'ils changent de sexe, d'abord femelles à la naissance puis deviennent mâles. Durant ces deux années d'étude (2006, 2007), l'étendue de la longueur obtenue dans la pêcherie était dans l'intervalle 10 $\mathrm{cm}-185 \mathrm{~cm}$, tandis que l'intervalle de taille qui a contribué de façon significative à la pêcherie était situé entre $35 \mathrm{~cm}-95 \mathrm{~cm}$. En tenant compte des travaux de Bou-Ain (1980) qui affirme que le mérou atteint sa maturité sexuelle à la taille de $40 \mathrm{~cm}$ et qu'à $50 \mathrm{~cm}$ toutes les femelles sont adultes, on en déduit que la majorité des individus pêchés sont des femelles. Cette thèse est soutenue par Gem (2006), qui a affirmé que les femelles changent de sexe vers 10-12 ans atteignant la taille d'environs $80 \mathrm{~cm}$, les individus mâles sont de grande taille, au delà de $90 \mathrm{~cm}$. Doris (2008) dans son article aborde dans le même sens en soutenant que les mérous deviennent des femelles capables de se reproduire vers l'âge de 4 ou 5 ans (40-50 cm environ). Entre 10 et 14 ans $(60-70 \mathrm{~cm})$, il se produit un changement de sexe. Celui-ci peut se produire plus tôt ou plus tard selon les individus, mais généralement, après 15 ans $(80-90 \mathrm{~cm})$ la majorité des individus sont devenus des mâles. Le mérou finit ainsi sa vie sous la forme d'un mâle, capable de se reproduire encore longtemps (Doris, 2008). Ainsi, apparaît 
t-il clairement une dominance d'individus femelles, ce qui pourrait menacer la pérennité de l'espèce. A l'absence de reproduction, la productivité des stocks baisserait, entrainant aussi la baisse du taux de protéines animales, singulièrement celle d'origine poissonnière disponibles. Des mesures de réglementation de la pêche s'imposent donc, afin de préserver ces espèces sur les côtes ivoiriennes.

Le couple taille poids dont les paramètres sont représentés dans le ableau I, montre que la croissance est allométrique minorante chez les cinq premières espèces de mérou car pour celles-ci, le taux d'allométrie est inférieur à 3 (b $=2,92$ pour $E$. aeneus, $\mathrm{b}=2,83$ pour $E$. caninus, $\mathrm{b}=2,81$ pour $E$. guaza, $\mathrm{b}=2,92$ pour $E$. marginatus et $\mathrm{b}=2,88$ pour $E$. alexandrinus). Cela se vérifie à travers les grandes tailles observées dans les débarquements. Aussi, existe-il de fortes corrélations entre le poids et la longueur totale vu les coefficients très élevés de $\mathrm{r}(\mathrm{r}$ $=0,89$ pour $E$. aeneus, $\mathrm{r}=0,90$ pour $E$. caninus, $\mathrm{r}=0,87$ pour $E$. guaza, $\mathrm{r}=0,82$ pour $E$. marginatus, $\mathrm{r}=0,90$ pour $E$. alexandrinus et $\mathrm{r}=0,73$ pour $E$. taeniops). Quant à E. taeniops, cette croissance allométrique est majorante $(b=3,11)$. Elle se confirme à travers deux individus recensés ayant eu une taille supérieur à 90 $\mathrm{cm}$. La majorité des individus pêchés pour cette espèce se situe dans la gamme de taille $34 \mathrm{~cm}$ à $59 \mathrm{~cm}$.

Pour toutes les représentations graphiques, les points expérimentaux s'ordonnent autour de la courbe théorique comme le démontrent les valeurs des coefficients de corrélation qui sont toutes élevées. C'est une justification de ce que ces différentes équations établies traduisent judicieusement l'existence de rapport entre les poids et les longueurs correspondantes, c'est-à-dire longueurs totales pour E. aeneus, E. caninus, $E$. guaza, E. marginatus, E. taeniops et longueur à la fourche pour $E$. alexandrinus.

\section{Conclusion}

$\mathrm{Au}$ terme de cette étude, la croissance d'E. aeneus, d'E. caninus, d'E. guaza, d'E. marginatus et d'E. alexandrinus est allométrique minorante tandis que celle d'E. taeniops est allométrique majorante.

\section{REFERENCES BIBLIOGRAPHIQUES}

Bou-Ain A. 1980. Sexualité et cycle sexuel des mérous (poissons téléostéens Serranidae) des côtes du sud tunisien. Bulloff. Nat. Pêch., 4(2): 215-229.

Champagnat, Domain F. 1978. Migrations des poissons démersaux le long des côtes ouest africaines de 10 à $24^{\circ}$ de latitude Nord. Cah. ORSTOM. Sér. Océanogr., 16: 239-261.

Dah A, Girardin M, Vall M. 1991. Les poissons de la communauté à Sciaenidés. In Bull. CNROP, 23. p.92.

Doris. 2008. Epinephelus marginatus (Low, 1834), http://doris.ffessm.fr/ fiche2.asp?fiche_numero $=474$, 13/9/2008.

Fischer W, Bianchi G, Scott WB. 1981. Fiches FAO d'Identification des Espèces pour les Besoins de la Pêche (Volume IV). $324 \mathrm{p}$. 
Gem. 2006. Recensement de la population de mérou brun (Epinephelus marginatus : Pisces) du Parc national de Port-Cros (France, Méditerranée) en 2005. Sci. Rep. Port-Cros Natl. Park, 22.

Hureau JC. 1986. Mullidae. in Fishes of the North-eastern Atlantic and the Mediterranean (Vol. 2). UNESCO: Paris; 877-882.

Laurans M. 2005. Ressources et exploitations «démersales» en Afrique de l'Ouest: évaluation des stocks, dynamique des populations et approche écosystémique. thèse de doctorat de l'école Nationale Sup. Agron. de Rennes, spécialité : Halieutique, pp.125, 161.

Layachi M, Melhoaoui M, Srour A, Ramdani M. 2007. Contribution à l'étude de la reproduction et de la croissance du Rouget barbet de vase (Mullus barbatus L., 1758) de la zone littorale méditerranéenne de Nador (Maroc). Bulletin de l'Institut Scientifique, Rabat, Section Sciences de la Vie, 29 : 43-51.

Le Cren ED. 1951. The length weight relationship and seasonal cycle in gonad weight and condition in the
Perch (P. fluviatilis). J. Anim. Ecol, 20: 201-219.

Moustapha OT. 2005. Etude des variations de la biomasse du thiof sur les côtes de Mauritanie. Influence de facteurs hydrodynamiques et climatiques. Laboratoire d'Evaluation des Stocks(LES) et Laboratoire d'Océanographie de Villefranche-surmer.30 p.

N'da K., Christian DL, et Yao K. 2006. Croissance du rouget de roche Mullus surmuletus dans le nord du golfe de Gascogne. Cybium, 30(1): 57-63.

N'da K., Kouassi KD, N'goran Y. 2006. Pêche artisanale maritime et commercialisation du mérou blanc (Epinephelus aeneus, Geoffroy Saint Hilaire, 1817) en Côte d'Ivoire. Tropicultura, 24:107-110

O.R.S.T.O.M Paris, 1981, réédition 1990. $449 \mathrm{p}$.

Opic P, Seret B. 1981. Poissons de mer de l'ouest africain tropical. IDT, ${ }^{\circ} 49$.

Wolfgand S. 1990. Guide de terrain des ressources marines commerciales du Golf de Guinée; Fiches FAO d'identification des espèces pour les besoins de la pêche, RAFR/FI/90/2 (F) Rome, 268 p. 